\title{
Shielding Effect of a Diaphragm in a Packaged Microstrip Circuit
}

\author{
Hao-Hui Chen and Shyh-Jong Chung, Member, IEEE
}

\begin{abstract}
The scattering of a diaphragm in a packaged microstrip line is investigated using the mode-matching method together with the method of lines. Instead of analyzing the discontinuity in the microstrip, this paper tackles the variation of the waveguide housing. The influences of the thickness and the depth of the diaphragm on the scatterings of the dominant mode and higher-order modes are analyzed based on a comparison of the modal power distributions. Finally, the shielding effect of a pair of closely spaced diaphragms is investigated.
\end{abstract}

\section{INTRODUCTION}

I $\mathrm{N}$ a (monolithic) microwave circuit, active devices or passive components radiate energy due to the discontinuities of the signal transmission lines. For an open-type circuit, the radiated power is carried by the surface waves and/or radiation waves of the substrate. For a packaged one, these waves change into the higher-order modes of the shielded transmission line. As these parasitic higher-order modes travel toward another device, the power is coupled into the transmission line through the second device, and the normal signal on the line is disturbed. Suitable methods should be adopted to guard devices from the electromagnetic interference (EMI) caused by these spurious modes. One of the approaches is to partition the packaged circuit into several cabinets using the metal diaphragms (Fig. 1).

The design of the metal diaphragms depends on how the modal fields are distributed over the cross section of the waveguide. For a shielded microstrip line, the higher-order modes have higher field density over the air region than has the dominant mode (microstrip mode). The dominant mode, which carries signals, has most of its field concentrated in the substrate region beneath the microstrip and part of the field distributed above the microstrip due to the fringing effect.

Essentially, the larger the depth of the diaphragm is, the greater is the power of the incident higher-order modes which can be reflected back, and thus the better is the isolation which can be achieved. But, as the depth is increased so that the diaphragm reaches the near zone of the microstrip, the field of the dominant mode is perturbed, causing a disruption of the signal. This implies that the depth of the diaphragm should be appropriately designed to get the maximum shielding effect, while not disturbing the signal.

Manuscript received June 14, 1994; revised September 15, 1994. This work was supported by the National Science Council of the R. O. C. under Grant NSC 82-0404-E-009-336.

The authors are with the Institute of Communication Engineering, National Chiao Tung University, Hsinchu, Taiwan, R.O.C

IEEE Log Number 9410331

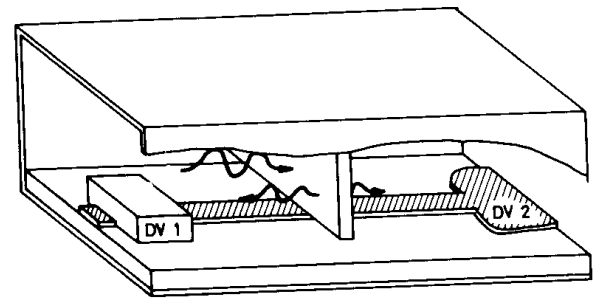

Fig. 1. A diaphragm in a shielded microstrip line.

The analysis of the scattering of a diaphragm in a shielded microstrip line is somewhat similar to that of an iris in a rectangular or cylindrical waveguides. For the scattering analysis of the iris, the mode-matching method has been used by many researchers to handle the discontinuity problems [1]-[4]. In their analyses, the structures of the waveguides were uniform, and the eigenmodes were easily found. The same method has also been adopted to tackle the step-type discontinuities in the shielded microstrip or other planar transmission lines [5]-[13]. Since the structures of the treated waveguides were complicated, numerical methods such as the spectral domain approach [5]-[9], the equivalent waveguide model [10], [11], and other approaches [12], [13], were used to obtain the field distributions of the eigenmodes. These eigenmodes may include complex modes [14], [15], which need a root searching in the complex plane. In general, only a small number of eigenmodes were required to expand the fields at the step due to the invariability of the waveguide housing.

In this paper the mode-matching method is used to analyze the shielding effect of a diaphragm inserted in a closed microstrip waveguide. The eigenmodes of each waveguide region are calculated by the method of lines $(\mathrm{MoL})$ [16]. Since the housings of the waveguides are different in each region, more eigenmodes are needed in the mode-matching procedure. The depth and the thickness of the diaphragm are varied to see the influences on the shielding effects with respect to the dominant and higher-order modes. Also, the shielding effects of a pair of closely spaced diaphragms are investigated.

\section{ANALYSIS}

Fig. 2 shows the structure for analysis, where a diaphragm with depth $d$ and thickness $t$ is attached to the top cover of a packaged microstrip waveguide. The microstrip is located symmetrically in the $x$ direction and has a width $(w)$ equal to the thickness $(h)$ of the substrate. The dimension of the housing is set to be $10 h \times 10 h$. Let the propagation modes of 

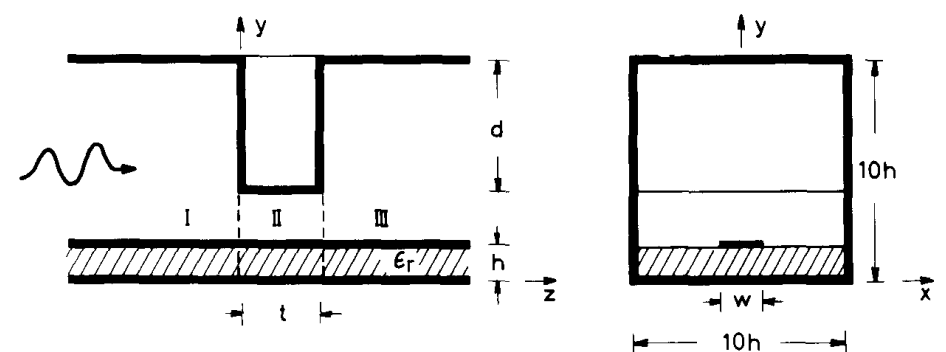

Fig. 2. Structure for analysis. $\epsilon_{r}=9.7, h=u=0.635 \mathrm{~mm}$.

the packaged microstrip waveguide be incident from the left of the diaphragm. These modes may be excited by some active device or passive component and may represent the signal (the dominant mode) or the parasitic wave (higher-order modes).

To tackle the problem, the modes, including propagation, evanescent, and complex, of the microstrip waveguides with the housings $10 h \times 10 h$ and $10 h \times(10 h-d)$ are first solved by the method of lines. Following the procedure described in [16], a homogeneous matrix equation is obtained

$$
[Z(\gamma)]\left[\begin{array}{l}
\mathbf{J}_{x} \\
\mathbf{J}_{z}
\end{array}\right]_{\text {strip }}=0
$$

where $\mathbf{J}_{x}$ and $\mathbf{J}_{z}$ are two vectors representing the $x$ and $z$ components of the current distributed on the strip, and $\gamma$ is the propagation constant of the mode, which may be imaginary (propagation modes), real (evanescent modes), or complex (complex modes).

To get nontrivial solutions of the current distributions, the determinant of the impedance matrix $[Z(\gamma)]$ should be zero, from which the propagation constants are obtained. In this analysis the $\gamma$ 's for the propagation modes are found by the Muller's method, while those for evanescent modes are obtained by searching in the negative real axis of the $\gamma$ plane for the sign changes of the determinant of $[Z(\gamma)]$. The required $\gamma$ 's (excluding the poles of the determinant) are first set as initial guesses, and the Muller's method is used to get more exact solutions. To find the $\gamma$ 's for the complex modes, the evanescent modes at a frequency several $\mathrm{GHz}$ apart from the operating frequency are first obtained. The frequency is then changed toward the operating one. At each sampled frequency, the propagation constants are searched for by the Muller's method with the propagation constants obtained at the previous sampled frequency as the initial guesses.

With $\gamma$ known, the current distributions on the strip are determined from (1), and all the fields of each mode are explicitly calculable. In this paper, a fine equal-distance discretization is used in the calculation to handle the large field variations of the highest-order modes. The fields of each mode are normalized so that its power equals 1 for a propagation mode, equals $\pm i$ $(i=\sqrt{(-1)})$ for an evanescent mode, and its absolute value equals unity for a complex mode.

After the eigen modes for each housing are found, the modematching method [2], [8], [10] is then adopted to determine the scattering matrix of the diaphragm. First, the computed modal fields are used to expand the fields at the two sides of the junction formed by the waveguides I and II (see Fig. 2). The continuities of the tangential electric and magnetic fields lead to two sets of equations, which are then suitably weighted by the modal fields [2], [10]. After this, a relation between the incident waves $\left(\mathbf{A}^{+}, \mathbf{B}^{-}\right)$and the scattered waves $\left(\mathbf{A}^{-}, \mathbf{B}^{+}\right)$ is obtained

$$
\left[\begin{array}{l}
\mathbf{A}^{-} \\
\mathbf{B}^{+}
\end{array}\right]=\left[\mathbf{S}_{\text {junc }}\right]\left[\begin{array}{l}
\mathbf{A}^{+} \\
\mathbf{B}^{-}
\end{array}\right]
$$

where $\mathbf{A}$ and $\mathbf{B}$ are vectors formed by the modal amplitudes of waveguides I and II, respectively. The superscripts " \pm " correspond to $\pm z$ propagations.

Once the scattering matrix $\mathbf{S}_{\text {junc }}$ of a single junction is determined, that of the diaphragms can be obtained by cascading the scattering matrices of all the junctions.

\section{NUMERICAL RESULTS}

In this paper we set $h=w=0.635 \mathrm{~mm}, \epsilon_{r}=9.7$, and the operating frequency $f=25 \mathrm{GHz}$. Under this choice of the parameters, there are three propagation modes for the microstrip waveguide with housing $10 h \times 10 h$, in which the first two (dominant mode and 2nd-order mode) are even modes and the last one (3rd-order mode) is an odd mode. In the method-of-lines calculation of the modal fields, the number of the e-lines passing through the microstrip is 20 , which has been shown to be sufficient for the present analysis. The validity of the obtained modes, especially for higher-order evanescent modes and complex modes, should be checked. This is done by satisfying the waveguide characteristic equation and all the boundary conditions. Also, the calculated modes satisfy the orthogonality relationships and the pair of complex modes satisfy the strong coupling and the orthogonality relationships referred to in [14]. Finally, the conservation and the convergence of the complex power, i.e., the average power and the reactive power, at the planes of $z=0$ and $z=t$ have been checked to further insure the validity of the works about the mode-matching method.

Since the design of the diaphragms depends on what are the field distributions of the waveguide modes, we compare the distributions of the $z$-propagated powers $\left(P_{z}\right.$ 's) for the three propagation modes of the $10 h \times 10 h$ waveguide. From the three-dimensional views of the power distributions, it has been found that the powers diverge at the strip edges and decay as the observation point leaves the edges. Moreover, the dominant mode has more power concentrated in the substrate region than 


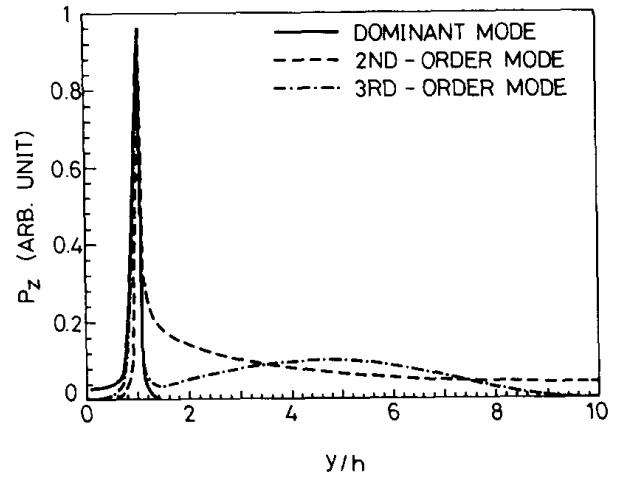

Fig. 3. $z$-propagated power distributions along the $y$ direction at $x=w / 2$ for the dominant and higher-order modes of the shielded microstrip line.

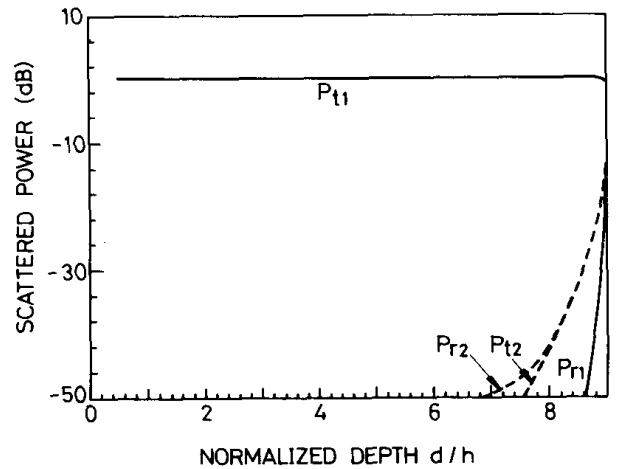

Fig. 4. Variation of the scattered powers as a function of the normalized diaphragm depth $(d / h)$ for a dominant-mode-incidence. $t=2.1 \mathrm{~mm}, f=$ $25 \mathrm{GHz}$.

do the 2nd-order mode and 3rd-order mode. Fig. 3 compares the power distributions along the $y$ direction at $x=w / 2$ for the three modes. Note that $y / h=1$ is the interface between the substrate and air regions. It is found that for the dominant mode, the power decays to almost zero at about $y / h=1.4$, but for the higher-order modes, a large part of the power is distributed in the air region. For the 2nd-order mode, although the power decays, there is still a notable value even at $y / h=$ 10 (the top cover of the waveguide). For the 3 rd-order mode, the power distribution does not decay monotonically but has a considerable value near $y=5 h$. This suggests that one may use a diaphragm with suitable depth to reflect the powers of the higher-order modes and not disturb the dominant mode's power.

The shielding effect of the diaphragms with different depths can be observed in Figs. 4-6. Fig. 4 presents the variations of the scattered powers as a function of the diaphragm depth for the dominant-mode incidence, and Figs. 5 and 6 present those for the 2nd-order-mode and 3rd-order-mode incidents, respectively. Here $P_{t i}\left(P_{r i}\right)$ represents the transmitted (reflected) power of the $i$ th mode. From Fig. 4 it is seen that increasing the depth of the diaphragm does not influence the propagation of the dominant mode. Even when the diaphragm is only $0.1 h$ above the microstrip $(d=8.9 h)$, the transmission

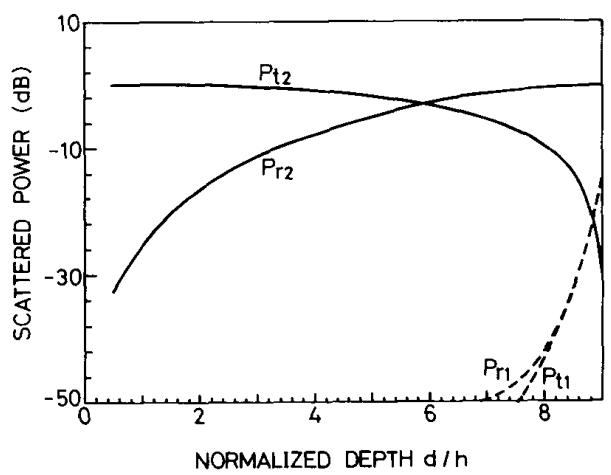

Fig. 5. Variation of the scattered powers as a function of the normalized diaphragm depth $(d / h)$ for a 2 nd-order-mode incidence. $t=2.1 \mathrm{~mm}, f=$ $25 \mathrm{GHz}$

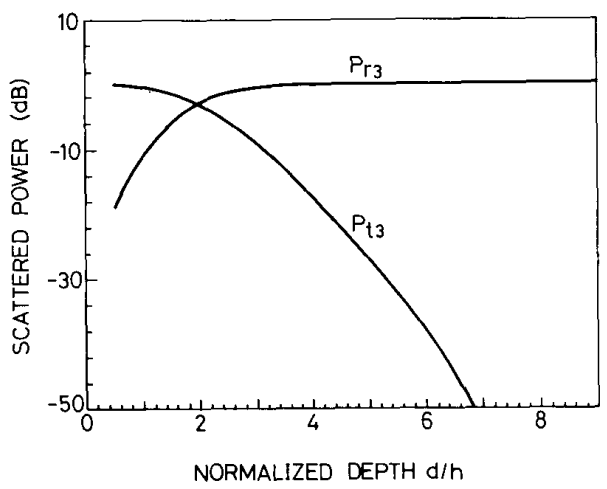

Fig. 6. Variation of the scattered powers as a function of the normalized diaphragm depth $(d / h)$ for a 3 rd-order-mode incidence. $t=2.1 \mathrm{~mm}, f=$ $25 \mathrm{GHz}$.

loss $\left(1 / P_{t 1}\right)$ is still less than $0.1 \mathrm{~dB}$. Nevertheless, the reflected power $\left(P_{r 1}\right)$ increases rapidly as the diaphragm is approaching the microstrip. In the limiting case in which the diaphragm touches the microstrip, a dc return path is formed and there may be more power being reflected back.

The scattering for the higher-order-mode incidents is quite different from that for the dominant-mode incidence. Large variations of the transmission $\left(P_{t 2}, P_{t 3}\right)$ and reflection $\left(P_{r 2}\right.$, $P_{r 3}$ ) powers due to the change of the diaphragm depth can be observed from Figs. 5 and 6 . For the 2nd-order-mode incidence, Fig. 5 shows that the depths $(d)$ for $3-, 10-$, and $20-\mathrm{dB}$ transmission losses $\left(1 / P_{t 2}\right)$ are $6 h, 8 h$, and $8.8 h$, respectively. The difference between the incident and transmitted powers is carried by the reflected 2 nd-order mode. The power coupled to the dominant mode $\left(P_{r 1}\right.$ and $\left.P_{t 1}\right)$ is negligible except when the diaphragm is very close to the microstrip. (For example, as $d=8.9 h$, both the transmitted and reflected dominant modes get one percent of the incident power.) Note that the two dashed lines $\left(P_{t 2}, P_{r 2}\right)$ in Fig. 4 are identical with those $\left(P_{t 1}, P_{r 1}\right)$ in Fig. 5 , which is the consequence of the reciprocity theorem. For the 3rd-order-mode incidence, the shielding effect is more obvious. From Fig. 6 it is seen that the depth $(d)$ for 3-, 10-, and 20-dB transmission losses $\left(1 / P_{t 3}\right)$ are $2 h, 3.2 h$, and $4.4 h$, respectively. This substantial 


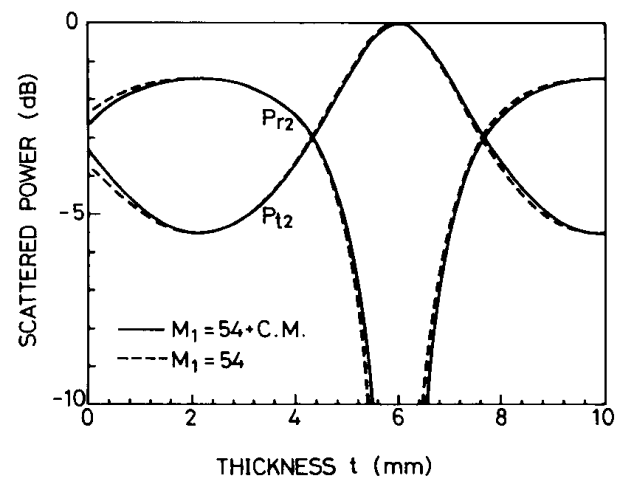

Fig. 7. Variation of the scattered powers as a function of the diaphragm thickness $(t)$ for a 2nd-order-mode incidence. $d=7 h, f=25 \mathrm{GHz}$.

scattering occurs because most of the power of the 3rd-order mode is concentrated near half of the waveguide height, and that the 3rd-order mode of waveguide II (the region beneath the diaphragm) becomes evanescent when $d>1.18 h$. In this case $(d>1.18 h)$, the incident power coming from waveguide I is coupled to waveguide III (Fig. 2) entirely by the evanescent fields in waveguide II. It is noticed that, since the 3rd-order mode is an odd mode but the dominant and 2nd-order modes are even, the incident power carried by the 3rd-order mode is not coupled to the dominant and 2nd-order modes. (In our numerical calculations the coupling powers between the odd and even modes are less than $-100 \mathrm{~dB}$.) Similarly, for the dominant-mode and the 2nd-order-mode incidents, there is no power transferred to the 3rd-order mode (as can be noticed in Figs. 4 and 5).

To find the influence of the diaphragm thickness on the shielding effect of the higher-order modes, Figs. 7 and 8 show the transmission $\left(P_{t 2}, P_{t 3}\right)$ and reflection $\left(P_{r 2}, P_{r 3}\right)$ powers for, respectively, an incident 2 nd-order mode and a 3rd-order mode. The depth $(d)$ of the diaphragm is set to be $7 h$. In Fig. 7, the dashed lines represent the results calculated using 54 expansion modes in waveguides I and III and 16 modes in waveguide II, both including only propagation and evanescent modes. The solid lines are the results with an extra pair of complex modes in each waveguide. The periodical behaviors of the curves come from the bouncing of the 2nd-order mode in waveguide II between the two junctions of the region beneath the diaphragm. The period $(7.6 \mathrm{~mm})$ is approximately equal to the half-wavelength $(7.73 \mathrm{~mm})$ of the 2 nd-order mode of waveguide II. It is seen that at thicknesses $t=2.1 \mathrm{~mm}$ and 9.7 $\mathrm{mm}$, one has the minimum transmission power $\left(P_{t 2}=-5.5\right.$ $\mathrm{dB})$. But, as the thickness is chosen to be $6 \mathrm{~mm}$, nearly all the incident power transmits through the diaphragm, which means that the diaphragm has no shielding effect on the incident wave. It is also noticed that the inclusion of the complex modes has influence on the calculated scattered powers only when the diaphragm is very thin $(t<0.4 \mathrm{~mm})$. This means that in practice $(t>0.5 \mathrm{~mm})$ the complex modes can be ignored. The complication of the analysis can thus be greatly reduced.

From Fig. 8 it is seen that, no matter what the thickness is, the power is almost entirely reflected back for a 3rd-order-

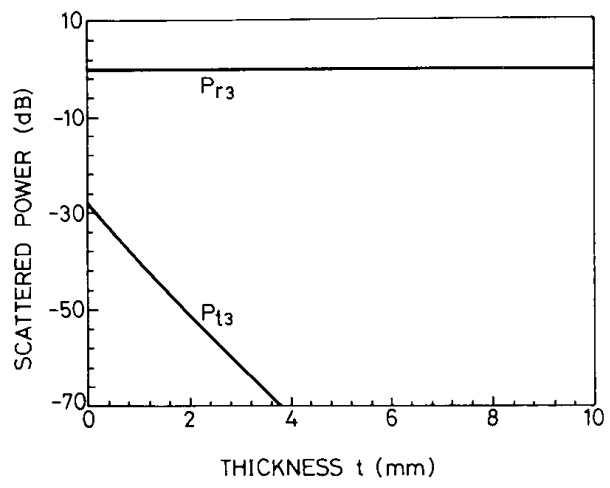

Fig. 8. Variation of the scattered powers as a function of the diaphragm thickness $(t)$ for a 3rd-order-mode incidence. $d=7 h, f=25 \mathrm{GHz}$.

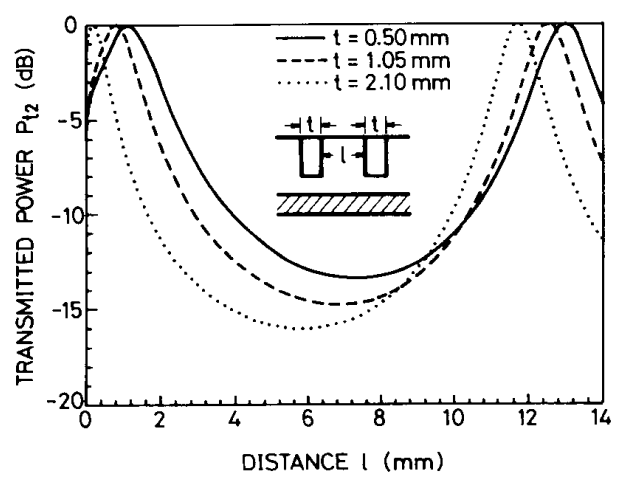

Fig. 9. Variation of the transmitted powers for a pair of diaphragms, as a function of the distance ( $l$ ) between the diaphragms. The incident wave is the 2nd-order of shielded microstrip line. $d=7 h, f=25 \mathrm{GHz}$.

mode incidence, as a result of the deep diaphragm $(d=$ $7 h$ ). Nevertheless, the transmission power $\left(P_{t 3}\right)$ decreases monotonically with the increase of the diaphragm thickness. It is noticed that, since the 3rd-order mode and the other odd modes in waveguide II are evanescent when $d=7 h$, no oscillations of the curves are observed.

Fig. 9 shows the transmitted power for a 2 nd-order-mode incident upon two diaphragms (both with depth $d=7 h$ ), as a function of the distance between the diaphragms. The introduction of the space between the diaphragms first increases the transmitted power to almost $0 \mathrm{~dB}$, then decreases it to a minimum value which is about $10 \mathrm{~dB}$ less than that of a single diaphragm with the same depth $(d=7 h)$ (see Fig. 7). The thicker are the diaphragms, the smaller are the minima. It is also seen that the resonant lengths for the diaphragms with thicknesses $t=0.50,1.05$, and $2.10 \mathrm{~mm}$ are the same $(l=$ $11.8 \mathrm{~mm}$ ), and are approximately equal to the half-wavelength $(11.03 \mathrm{~mm})$ of the 2 nd-order mode.

\section{CONCLUSION}

The shielding effect of a diaphragm in a housed microstrip waveguide has been analyzed using the mode-matching method together with the method of lines. In the modematching procedure, ignoring the complex modes of the 
shielded lines causes numerical errors only when the diaphragm is very thin $(<0.4 \mathrm{~mm})$. It has been shown that the thickness of the diaphragm should be carefully designed to obtain the optimum shielding effect. Also, by increasing the depth, the diaphragm can reflect almost all the power of the incident higher-order modes while it has little influence on the propagation of the dominant mode. Finally, the shielding effect of a pair of diaphragms has been found to be about $10 \mathrm{~dB}$ better than that of a single one, with the diaphragms being suitably spaced.

\section{REFERENCES}

[1] R. E. Collin, Field Theory of Guided Waves, 2nd ed. New York: IEEE Press, 1991.

[2] A. Wexler, "Solution of waveguide discontinuities by modal analysis," IEEE Trans. Microwave Theory Tech., vol. MTT-15, pp. 508-517, Sept. 1967.

[3] R. Safavi-Naini and R. H. MacPhie, "On solving waveguide junction scattering problems by the conservation of complex power technique," IEEE Trans. Microwave Theory Tech., vol. MTT-29, pp. 337-343, Apr. 1981.

[4] J. D. Wade and R. H. MacPhie, "Scattering at circular-to-rectangular waveguide junctions," IEEE Trans. Microwave Theory Tech., vol. MTT34, pp. 1085-1091, Nov. 1986.

[5] C.-W. Kuo and T. Itoh, "Characterization of shielded coplanar type transmission line junction discontinuities incorporating the finite metallization thickness effect," IEEE Trans. Microwave Theory Tech., vol. MTT-40, pp. 73-80, Jan. 1992

[6] Q. Xu, K. J. Webb, and R. Mittra, "Study of modal solution procedures for microstrip step discontinuities," IEEE Trans. Microwave Theory Tech., vol, MTT-37, pp. 381-386, Feb. 1989.

[7] O. Picon, V. F. Hanna, J. Citerne, and J.-P. Lefevre, "Exact calculation of scattering parameters of the coplanar-slot transition in unilateral finline technology," IEEE Trans. Microwave Theory Tech., vol. MTT-35, pp. 1408-1413, Dec. 1987

[8] M. Helard, J. Citerne, O. Picon, and V. F. Hanna, "Theoretical and experimental investigation of finline discontinuities," IEEE Trans. Microwave Theory Tech., vol. MTT-33, pp. 994-1003, Oct. 1985.

[9] N. H. L. Koster and R. H. Jansen, "The microstrip step discontinuity: A revised description," IEEE Trans. Microwave Theory Tech., vol. MTT34, pp. 213-222, Feb. 1986.

[10] T. S. Chu, T. Itoh, and Y.-C. Shih, "Comparative study of modematching formulations for microstrip discontinuity problems," IEEE Trans. Microwave Theory Tech., vol. MTT-33, pp. 1018-1023, Oct. 1985.
[11] T. S. Chu and T. Itoh, "Generalized scattering matrix method for analysis of cascaded and offset microstrip step discontinuities," IEEE Trans. Microwave Theory Tech., vol. MTT-34, pp. 280-284, Feb. 1986.

[12] N. K. Uzunoglu, C. N. Capsalis, and C. P. Chronopoulos, "Frequencydependent analysis of a shielded microstrip step discontinuity using an efficient mode-matching technique," IEEE Trans. Microwave Theory Tech., vol. MTT-36, pp. 976-984, June 1988.

[13] K. J. Webb and R. Mittra, "Solution of the finline step-discontinuity problem using the generalized variational technique," IEEE Trans Microwave Theory Tech., vol. MTT-33, pp. 1004-1010, Oct. 1985.

[14] A. S. Omar and K. Schünemann, "Formulation of the singular integral equation technique for planar transmission lines," IEEE Trans. Microwave Theory Tech., vol. MTT-33, pp. 1313-1322, Dec. 1985.

[15] W.-X. Huang and T. Itoh, "Complex modes in lossless shielded microstrip lines," IEEE Trans. Microwave Theory Tech., vol. MTT-36, pp. 163-165, Jan. 1988.

[16] R. Pregla and W. Pascher, "The method of lines," in Numerical Techniques for Microwave and Millimeter Wave Passive Structures, T. Itoh, Ed. New York: Wiley, 1989, ch. 6.

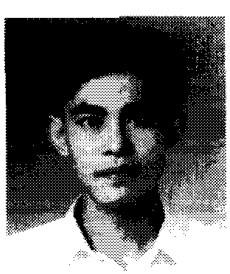

Hao-Hui Chen was born in Kaohsiung, Taiwan, R. O. C. on April 11, 1969. He received the B.S. degree in physics from the National Central University, Chung-Li, Taiwan, in 1991. Since 1992, he has been studying toward the Ph.D. degree in communication engineering at National Chiao Tung University, Hsinchu, Taiwan, R.O.C

His research interest include electromagnetic theory, antenna and waveguide analysis, and numerical techniques in electromagnetics.

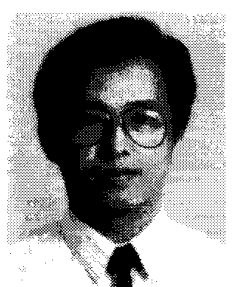

Shyh-Jong Chung (M'92) was born in Taipei, Taiwan, R. O. C., on January 18, 1962. He received the B.S.E.E. degree in 1984 and the Ph.D. degree in 1988, both from the National Taiwan University, Taipei, Taiwan.

Since 1988, he has been with the Department of Communication Engineering, National Chiao Tung University, Hsinchu, Taiwan, R.O.C., where he is currently an associate professor. His areas of interest include propagation and scattering of transmission lines, packaging effects of microwave circuits, numerical techniques in electromagnetics, and microstrip patch antennas. 